\title{
The Simultaneous Interpolation of Target Radar Cross Section in Both the Spatial and Frequency Domains by Means of Legendre Wavelets Model-Based Parameter Estimation
}

\author{
Yongqiang Yang, Yunpeng Ma, and Lifeng Wang \\ School of Aeronautic Science and Technology, Beihang University, Beijing 100191, China \\ Correspondence should be addressed to Yunpeng Ma; yunpengma@163.com
}

Received 12 January 2015; Revised 17 May 2015; Accepted 18 May 2015

Academic Editor: Mahmut Reyhanoglu

Copyright (c) 2015 Yongqiang Yang et al. This is an open access article distributed under the Creative Commons Attribution License, which permits unrestricted use, distribution, and reproduction in any medium, provided the original work is properly cited.

\begin{abstract}
The understanding of the target radar cross section (RCS) is significant for target identification and for radar designing and optimization. In this paper, a numerical algorithm for calculating target RCS is presented which is based on Legendre wavelet model-based parameter estimation (LW-MBPE). The Padé rational function fitting model applied for MBPE in the frequency domain is enhanced to include spatial dependence on the numerator and denominator coefficients. This allows the function to interpolate target RCS in both the frequency and spatial domains simultaneously. The combination of Legendre wavelets guarantees the convergence of the algorithm. The method is convergent by increasing the sampling frequency and spatial points. Numerical results are provided to demonstrate the validity and applicability of the new technique.
\end{abstract}

\section{Introduction}

In modern electronic warfare, stealth technology is the main technique used to reduce radar detection probability and enhance the survivability of aircrafts $[1,2]$. RCS reduction is the key factor to measure stealth performance of the aircraft. RCS reduction techniques of aircraft generally fall into one of four categories $[3,4]$ : materials selection and coating, target shaping, passive cancellation, and active cancellation. Active cancellation stealth is a significant research direction in the field of stealth. The creation of a large RCS database of target is the key process in active cancellation [5-7]. However, although the parallel technology of computer is rapidly developing, it is still an arduous task to create a large RCS database containing both frequency and spatial domain information. In recent years, the model-based parameter estimation (MBPE) [8-10] is combined with the method of moments (MoM) to minimize the computational cost. This method is widely used in solving the calculation of target RCS problems $[11,12]$. Since it includes the frequency and spatial domain information, it is also used to store and predict target RCS and create RCS database. A lot of articles describe in detail the theory behind the MBPE interpolation process $[13,14]$. In [12], the modeling, sampling, and solution of MBPE for both frequency and domain problems are described.

Wavelet analysis is a new and an emerging area in engineering and mathematical research [15]. Wavelets are used in optimal control, system analysis, signal analysis, numerical analysis, and fast algorithms for easy implementation. Functions are decomposed into summation of "basic functions," and every "basic function" is achieved by compression and translation of a mother wavelet function with good properties of smoothness and locality, which makes people analyze the properties of locality and integer in the process of expressing functions $[16,17]$.

In this work, a numerical method based on the Legendre wavelets MBPE is proposed to compute target RCS approximately. A generalized Padé rational function fitting model that can be used to interpolate both frequency and spatial characteristics of RCS simultaneously is enhanced. Convergence analysis of the Legendre wavelets MBPE is 
investigated. Numerical results demonstrate the efficiency of this method in solving target RCS.

\section{Legendre Wavelets}

The Legendre wavelets $\psi_{n m}(x)$ are expressed as follows [18, 19]:

$$
\begin{aligned}
& \psi_{n m}(x) \\
& = \begin{cases}\left(\frac{2 m+1}{2}\right)^{1 / 2} 2^{k / 2} P_{m}\left(2^{k} x-\widehat{n}\right), & \frac{\widehat{n}-1}{2^{k}} \leq x<\frac{\widehat{n}+1}{2^{k}} \\
0, & \text { otherwise, }\end{cases}
\end{aligned}
$$

where $k=1,2, \ldots, \widehat{n}=2 n-1, n=1,2, \ldots, 2^{k-1}, m=$ $0,1, \ldots, M-1$ is the degree of the Legendre polynomials, $M$ is a fixed positive integer, and $P_{m}(x)$ are the Legendre polynomials of degree $m$.

For any function $f(x) \in L^{2}[0,1)$ may be given by the Legendre wavelets as

$$
f(x)=\sum_{n=1}^{\infty} \sum_{m=0}^{\infty} c_{n m} \psi_{n m}(x)
$$

where $c_{n m}=\left\langle f(x), \psi_{n m}(x)\right\rangle$ and $\langle$,$\rangle is the inner product of$ $f(x)$ and $\psi_{n m}(x)$.

If the infinite series in (2) is truncated, then we have

$$
f(x) \approx \sum_{n=1}^{2^{k-1}} \sum_{m=0}^{M-1} c_{n m} \psi_{n m}(x)=C^{T} \Psi(x)
$$

where $C$ and $\Psi(x)$ are $\widehat{m}=2^{k-1} M$ column vectors:

$$
\begin{aligned}
& C=\left[c_{10}, c_{11}, \ldots, c_{1 M-1}, c_{20}, c_{21}, \ldots, c_{2 M-1}, \ldots, c_{2^{k-1} 0},\right. \\
& \left.c_{2^{k-1} 1}, \ldots, c_{2^{k-1} M-1}\right]^{T} \\
& \Psi(x)=\left[\psi_{10}, \psi_{11}, \ldots, \psi_{1 M-1}, \psi_{20}, \psi_{21}, \ldots, \psi_{2 M-1}, \ldots,\right. \\
& \left.\psi_{2^{k-1} 0}, \psi_{2^{k-1} 1}, \ldots, \psi_{2^{k-1} M-1}\right]^{T} .
\end{aligned}
$$

For simplicity, we write (3) as

$$
f(x) \approx \sum_{i=1}^{\widehat{m}} c_{i} \psi_{i}(x)=C^{T} \Psi(x)
$$

where $c_{i}=c_{n m}, \psi_{i}=\psi_{n m}$. The index $i$ is determined by the relation $i=M(n-1)+m+1$. Therefore, we can also write the vectors

$$
\begin{aligned}
& C=\left[c_{1}, c_{2}, \ldots, c_{M}, c_{M+1}, \ldots, c_{2 M}, \ldots, c_{M\left(2^{k-1}-1\right)+1}, \ldots,\right. \\
& \left.c_{\widehat{m}}\right]^{T}, \\
& \Psi(x)=\left[\psi_{1}, \psi_{2}, \ldots, \psi_{M}, \psi_{M+1}, \ldots, \psi_{2 M}, \ldots,\right. \\
& \left.\quad \psi_{M\left(2^{k-1}-1\right)+1}, \ldots, \psi_{\widehat{m}}\right]^{T} .
\end{aligned}
$$

Similarly, for the two variables, function $u(x, y)$ defined over $[0,1) \times[0,1)$ may be expressed as the Legendre wavelets basis:

$$
u(x, y) \approx \sum_{i=1}^{\widehat{m}} \sum_{j=1}^{\widehat{m}} u_{i j} \psi_{i}(x) \psi_{j}(y)=\Psi^{T}(x) U \Psi(y)
$$

where $U=\left[u_{i j}\right]$ and $u_{i j}=\left\langle\psi_{i}(x),\left\langle u(x, y), \psi_{j}(y)\right\rangle\right\rangle$.

\section{Legendre Wavelets Model-Based Parameter Estimation Method}

The Padé rational function in the form of a fractional polynomial function of the $n$ order numerator and the $d$ order denominator employed commonly in MBPE is given by

$$
\begin{aligned}
F(s) & =\frac{N_{n}(s)}{D_{d}(s)} \\
& =\frac{N_{0}+N_{1} s+N_{2} s^{2}+\cdots+N_{n} s^{n}}{D_{0}+D_{1} s+D_{2} s^{2}+\cdots+D_{d-1} s^{d-1}+s^{d}},
\end{aligned}
$$

where $F(s)$ represents a frequency domain fitting model appropriate for the set of complex data and $s$ represents the complex frequency $j \omega=j 2 \pi f$, where $f$ is the frequency of interest. The function has $n+d+1$ unknown complex coefficients. To obtain accurate spatial resolution, the number of separate interpolations required and the overall number of resulting interpolation coefficients become very large. Therefore, we may write (8) in the more general form

$$
\begin{aligned}
& F(\theta, s)=\frac{N(\theta, s)}{D(\theta, s)} \\
& =\frac{N_{0}(\theta)+N_{1}(\theta) s+N_{2}(\theta) s^{2}+\cdots+N_{n}(\theta) s^{n}}{D_{0}(\theta)+D_{1}(\theta) s+D_{2}(\theta) s^{2}+\cdots+D_{d-1}(\theta) s^{d-1}+s^{d}},
\end{aligned}
$$

where

$$
\begin{aligned}
& N_{0}(\theta)=N_{0}^{0}+N_{0}^{1} \theta+N_{0}^{2} \theta^{2}+\cdots+N_{0}^{\widehat{k}} \theta^{\widehat{k}}, \\
& N_{1}(\theta)=N_{1}^{0}+N_{1}^{1} \theta+N_{1}^{2} \theta^{2}+\cdots+N_{1}^{\widehat{k}} \theta^{\widehat{k}},
\end{aligned}
$$

$$
\begin{aligned}
& N_{n}(\theta)=N_{n}^{0}+N_{n}^{1} \theta+N_{n}^{2} \theta^{2}+\cdots+N_{n}^{\widehat{k}} \theta^{\hat{k}}, \\
& D_{0}(\theta)=D_{0}^{0}+D_{0}^{1} \theta+D_{0}^{2} \theta^{2}+\cdots+D_{0}^{\widehat{k}} \theta^{\widehat{k}}, \\
& D_{1}(\theta)=D_{1}^{0}+D_{1}^{1} \theta+D_{1}^{2} \theta^{2}+\cdots+D_{1}^{\widehat{k}} \theta^{\widehat{k}},
\end{aligned}
$$


where $\widehat{k}$ represents the polynomial order of each coefficient. In (9), the $n+d+1$ unknown numerator and denominator coefficients now possess dependence on a spatial variable $\theta$. Thus (9) can be used to interpolate target radar cross section (RCS) as a function of both frequency and angle approximately. There are several possible models, which could be adopted to solve the coefficients $N_{i}^{j}$ and $D_{i}^{j}$. In this paper, we apply Legendre wavelets coefficients to approximate the coefficients $N_{i}^{j}$ and $D_{i}^{j}$.

$$
\begin{aligned}
& \mathbf{a}_{i}=\left[\begin{array}{cccccccc}
1 & s_{1} & \cdots & s_{1}^{n} & -F\left(\theta_{i}, s_{1}\right) & -F\left(\theta_{i}, s_{1}\right) s_{1} & \ldots & -F\left(\theta_{i}, s_{1}\right) s_{1}^{d-1} \\
1 & s_{2} & \cdots & s_{2}^{n} & -F\left(\theta_{i}, s_{2}\right) & -F\left(\theta_{i}, s_{2}\right) s_{2} & \cdots & -F\left(\theta_{i}, s_{2}\right) s_{2}^{d-1} \\
\vdots & \vdots & \ddots & \vdots & \vdots & \vdots & \ddots & \vdots \\
1 & s_{n+d+1} & \cdots & s_{n+d=1}^{n} & -F\left(\theta_{i}, s_{n+d+1}\right) & -F\left(\theta_{i}, s_{n+d+1}\right) s_{n+d+1} & \cdots & -F\left(\theta_{i}, s_{n+d+1}\right) s_{n+d+1}^{d-1}
\end{array}\right], \\
& \mathbf{b}_{i}=\left[\begin{array}{c}
F\left(\theta_{i}, s_{1}\right) s_{1}^{d} \\
F\left(\theta_{i}, s_{2}\right) s_{2}^{d} \\
\vdots \\
F\left(\theta_{i}, s_{n+d+1}\right) s_{n+d+1}^{d}
\end{array}\right] \\
& \mathbf{x}_{i}=\left[\begin{array}{c}
N_{0}\left(\theta_{i}\right) \\
N_{1}\left(\theta_{i}\right) \\
\vdots \\
N_{n}\left(\theta_{i}\right) \\
D_{0}\left(\theta_{i}\right) \\
D_{1}\left(\theta_{i}\right) \\
\vdots \\
D_{d-1}\left(\theta_{i}\right)
\end{array}\right] .
\end{aligned}
$$

$\mathbf{x}_{i}(i=1,2, \ldots, \widehat{k})$ will be got by solving (11).

Substituting $\mathbf{x}_{i}$ into (9), we have

$$
\begin{aligned}
& F\left(\theta_{i}, s\right)=\frac{N\left(\theta_{i}, s\right)}{D\left(\theta_{i}, s\right)} \\
& =\frac{N_{0}\left(\theta_{i}\right)+N_{1}\left(\theta_{i}\right) s+N_{2}\left(\theta_{i}\right) s^{2}+\cdots+N_{n}\left(\theta_{i}\right) s^{n}}{D_{0}\left(\theta_{i}\right)+D_{1}\left(\theta_{i}\right) s+D_{2}\left(\theta_{i}\right) s^{2}+\cdots+D_{d-1}\left(\theta_{i}\right) s^{d-1}+s^{d}} .
\end{aligned}
$$

For arbitrary $s_{l}$, we use Legendre wavelets method to obtain function $F\left(\theta, s_{l}\right)$, which is expressed as

$$
F\left(\theta^{\prime}, s_{l}\right) \approx \sum_{n=1}^{2^{k-1}} \sum_{m=0}^{M-1} c_{n m} \psi_{n m}\left(\theta^{\prime}\right),
$$

where $\theta^{\prime}=2 \theta / \pi$. Due to the arbitrariness of $s_{l}$, we can acquire the function $F(\theta, s)$ approximately.
By sampling the set of measured or calculated complex target RCS at $n+d+1$ frequency points and at $\hat{k}$ points in space, the expression in (9) can be written as partitioned matrix equations of the form

$$
\mathbf{a}_{i} \mathbf{x}_{i}=\mathbf{b}_{i}, \quad(i=1,2, \cdots, \widehat{k}),
$$

where

In this part, in order to illustrate the effectiveness of (14), we have given the following theorem. Let $F_{k, M}\left(\theta^{\prime}, s_{l}\right)$ be the following approximation of $F\left(\theta^{\prime}, s_{l}\right)$ :

$$
F_{k, M}\left(\theta^{\prime}, s_{l}\right)=\sum_{n=0}^{2^{k}-1} \sum_{m=0}^{M-1} c_{n m} \psi_{n m}\left(\theta^{\prime}\right)
$$

Then we have $F\left(\theta^{\prime}, s_{l}\right)-F_{k, M}\left(\theta^{\prime}, s_{l}\right)=$ $\sum_{n=2^{k}}^{+\infty} \sum_{m=M}^{+\infty} c_{n m} \psi_{n m}\left(\theta^{\prime}\right)$.

Theorem 1. Suppose that the function $F_{k, M}\left(\theta^{\prime}, s_{l}\right)$ obtained by using Legendre wavelets is the approximation of $F\left(\theta^{\prime}, s_{l}\right)$, and $F\left(\theta^{\prime}, s_{l}\right)$ is with bounded second derivative; then one has the following upper bound of error: 


$$
\begin{aligned}
& \left\|F\left(\theta^{\prime}, s_{l}\right)-F_{k, M}\left(\theta^{\prime}, s_{l}\right)\right\|_{E} \\
& \quad \leq\left(\frac{\widetilde{M}^{2}}{31} \frac{1}{2^{5 k+3}}\left(\frac{\Gamma^{\prime}(M-1.5)}{\Gamma(M-1.5)}\right)^{\prime \prime \prime}\right)^{1 / 2},
\end{aligned}
$$

where $\left\|F\left(\theta^{\prime}, s_{l}\right)\right\|_{E}=\left(\int_{0}^{1} F^{2}\left(\theta^{\prime}, s_{l}\right) d \theta^{\prime}\right)^{1 / 2}, c_{n m}=\left\langle F\left(\theta^{\prime}, s_{l}\right)\right.$, $\left.\psi_{n m}\left(\theta^{\prime}\right)\right\rangle$, and $\langle$,$\rangle is inner product of F\left(\theta^{\prime}, s_{l}\right)$ and $\psi_{n m}\left(\theta^{\prime}\right)$. $\Gamma^{\prime}\left(\theta^{\prime}\right) / \Gamma\left(\theta^{\prime}\right)$ is double gamma function.

Proof. See Appendix A.

From this theorem, we can see clearly that $\| F\left(\theta^{\prime}, s_{l}\right)-$ $F_{k, M}\left(\theta^{\prime}, \mathrm{s}_{l}\right) \|_{E} \rightarrow 0$ when $M$ is fixed and $k \rightarrow+\infty$.

The fitting model proposed in (9) may be extended to include target RCS which not only have a dependence on $\theta$, but also vary with $\varphi$. The general form of the fitting model under these conditions will be given as

$$
\begin{aligned}
& F(\theta, \varphi, s)=\frac{N(\theta, \varphi, s)}{D(\theta, \varphi, s)} \\
& =\frac{N_{0}(\theta, \varphi)+N_{1}(\theta, \varphi) s+N_{2}(\theta, \varphi) s^{2}+\cdots+N_{n}(\theta, \varphi) s^{n}}{D_{0}(\theta, \varphi)+D_{1}(\theta, \varphi) s+D_{2}(\theta, \varphi) s^{2}+\cdots+D_{d-1}(\theta, \varphi) s^{d-1}+s^{d}}
\end{aligned}
$$

where

$$
\begin{aligned}
& N_{i}(\theta, \varphi)=\sum_{j=1}^{p_{n}+1} \sum_{k=1}^{j} N_{i}^{(j-k, k-1)} \theta^{j-k} \varphi^{k-1}, \\
& D_{i}(\theta, \varphi)=\sum_{m=1}^{p_{n}+1} \sum_{n=1}^{m} N_{i}^{(m-n, n-1)} \theta^{m-n} \varphi^{n-1},
\end{aligned}
$$

where $p_{n}$ is the class of the binomials or the highest power of $\theta$ and $\varphi$ present in the binomial expansion.

As in the previous case, the Padé rational function defined by (17) is expanded using the set of coefficients given in (18) and then sampled at the appropriate number of data points in order to construct matrix equations of the form (11). Then (13) can be transformed into

$$
F\left(\theta_{i}, \varphi_{j}, s\right)=\frac{N\left(\theta_{i}, \varphi_{j}, s\right)}{D\left(\theta_{i}, \varphi_{j}, s\right)}=\frac{N_{0}\left(\theta_{i}, \varphi_{j}\right)+N_{1}\left(\theta_{i}, \varphi_{j}\right) s+N_{2}\left(\theta_{i}, \varphi_{j}\right) s^{2}+\cdots+N_{n}\left(\theta_{i}, \varphi_{j}\right) s^{n}}{D_{0}\left(\theta_{i}, \varphi_{j}\right)+D_{1}\left(\theta_{i}, \varphi_{j}\right) s+D_{2}\left(\theta_{i}, \varphi_{j}\right) s^{2}+\cdots+D_{d-1}\left(\theta_{i}, \varphi_{j}\right) s^{d-1}+s^{d}}
$$

Similarly, for arbitrary $s_{l}$, we apply Legendre wavelets method to get function $F\left(\theta, \varphi, s_{l}\right)$, which is given by

$$
F\left(\theta, \varphi, s_{l}\right) \approx \sum_{i=1}^{\widehat{m}} \sum_{j=1}^{\widehat{m}} u_{i j} \psi_{i}(\theta) \psi_{j}(\varphi)
$$

Due to the arbitrariness of $s_{l}$, we can obtain the function $F(\theta, \varphi, s)$ approximately.

Next, we will discuss the effectiveness of $F\left(\theta, \varphi, s_{l}\right) \approx$ $\sum_{i=1}^{\widehat{m}} \sum_{j=1}^{\widehat{m}} c_{i j} \psi_{i}(\theta) \psi_{j}(\varphi)$; we have given Theorem 2 . Let $F_{\widehat{m}}\left(\theta, \varphi, s_{l}\right)$ be the following approximation of $F\left(\theta, \varphi, s_{l}\right)$ :

$$
F_{\widehat{m}}\left(\theta, \varphi, s_{l}\right)=\sum_{i=1}^{\widehat{m}} \sum_{j=1}^{\widehat{m}} c_{i j} \psi_{i}(\theta) \psi_{j}(\varphi)
$$

Then we have $F\left(\theta, \varphi, s_{l}\right)-F_{\widehat{m}}\left(\theta, \varphi, s_{l}\right)$ $\sum_{i=\widehat{m}+1}^{\infty} \sum_{j=\widehat{m}+1}^{\infty} u_{i j} \psi_{i}(\theta) \psi_{j}(\varphi)$.

Theorem 2. Suppose that the function $F_{\widehat{m}}\left(\theta, \varphi, s_{l}\right)$ obtained by using Legendre wavelets is the approximation of $F\left(\theta, \varphi, s_{l}\right)$, and $F\left(\theta, \varphi, s_{l}\right)$ has bounded mixed fractional partial derivative $\left|\partial^{4} F\left(\theta, \varphi, s_{l}\right) / \partial \theta^{2} \partial \varphi^{2}\right| \leq \widehat{M}$; then one has the following upper bound of error:

$$
\left\|F\left(\theta, \varphi, s_{l}\right)-F_{\widehat{m}}\left(\theta, \varphi, s_{l}\right)\right\|_{E} \leq \frac{\widehat{M} \cdot \widehat{N}^{1 / 2}}{2^{4 k}},
$$

where $\left\|F\left(\theta, \varphi, s_{l}\right)\right\|_{E}=\left(\int_{0}^{1} \int_{0}^{1} F^{2}\left(\theta, \varphi, s_{l}\right) d \theta d \varphi\right)^{1 / 2}, u_{i j}=$ $\left\langle\psi_{i}(\theta),\left\langle F\left(\theta, \varphi, s_{l}\right), \psi_{j}(\varphi)\right\rangle\right\rangle$, and $\widehat{N}$ is a constant.
Proof. See Appendix B.

From this theorem, we can see that $\| F\left(\theta, \varphi, s_{l}\right)-F_{\widehat{m}}(\theta, \varphi$, $\left.s_{l}\right) \|_{E} \rightarrow 0$ when $k \rightarrow+\infty$.

\section{Numerical Results}

RCS, as understood in this paper, will represent the reflective strength of a radar target. RCS, denoted by the Greek letter $\sigma$ and measured in $\mathrm{m}^{2}$, is defined as [20]

$$
\sigma=4 \pi \frac{P_{s}}{P_{i}} .
$$

RCS has a wide spread ranging from $10^{-5}$ for small insects to $10^{6}$ for large targets. Hence, RCS is often expressed as the logarithmic decibel scale:

$$
\sigma_{\mathrm{dBsqm}}=10 \log \left(\frac{\sigma}{1 \cdot m^{2}}\right)
$$

the unit of (24) is $\mathrm{dB}$ (decibel).

The LW-MBPE technique described in the above section was first applied to an elliptical cylinder (Figure 1) over a frequency range of $0.5-2 \mathrm{GHz}$. The symmetry of this problem may be investigated such that it is only necessary to use the interpolation over the limited range $0^{\circ} \leq \theta \leq 90^{\circ}$. The Padé rational function was chosen to have a numerator order $n=8$ and a denominator order $n=7$. The fitting frequencies selected were $0.5,0.6,0.7, \ldots, 1.9$ and $2 \mathrm{GHz}$. These conditions were used to construct a matrix of the form given in (11), where $n+d+1=16$. The nonnormal incidence 


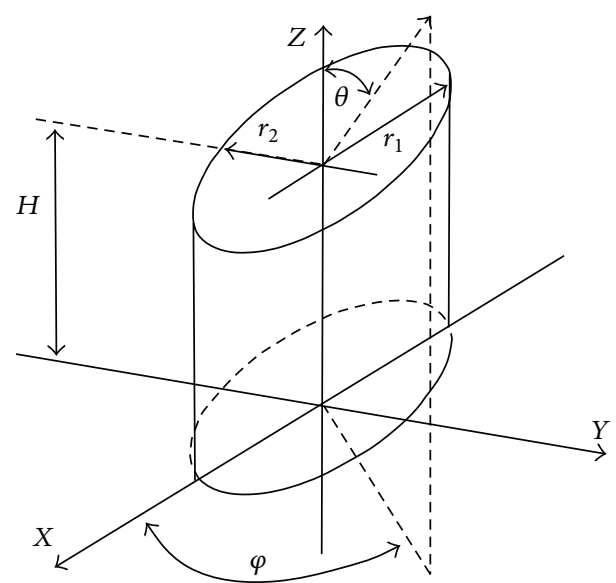

FIgURE 1: Elliptical cylinder.

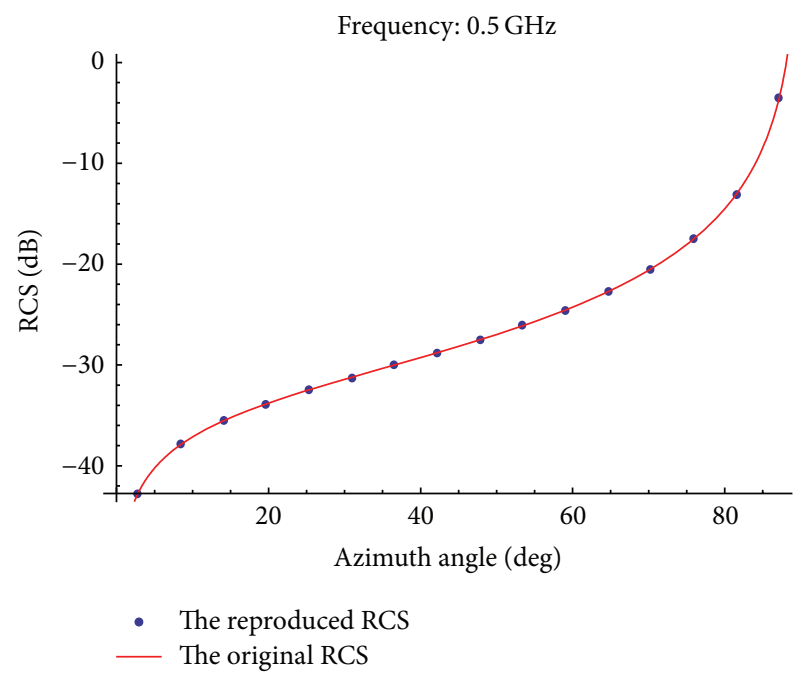

FIGURE 2: Backscattered RCS for an elliptical cylinder of $k=4$ and $\varphi=45^{\circ}$.

backscattered RCS for an elliptical cylinder due to a linearly polarized incident wave is given by [20]

$$
\sigma=\frac{\lambda r_{2}^{2} r_{1}^{2} \sin \theta}{8 \pi(\cos \theta)^{2}\left[r_{1}^{2}(\cos \varphi)^{2}+r_{2}^{2}(\sin \varphi)^{2}\right]^{1.5}}
$$

Figures 2, 3, 4, and 5 show the elliptical cylinder $\left(r_{1}=\right.$ $0.125 \mathrm{~m}, r_{2}=0.05 \mathrm{~m}$ ) backscattered RCS using (25) and the reproduced RCS using LW-MBPE method for different $k, M=2$. The absolute errors for the reproduced RCS and original RCS in Figures 2-5 are shown in Figure 6. From Figure 6, we can find easily that the absolute errors are rather small.

Table 1 shows the absolute errors between the original RCS and the reproduced RCS. From Table 1, we can also see that the errors are smaller and smaller when $k$ increases.

The interpolated RCS as a function of azimuth angle $\theta$ and pitching angle $\varphi$ is shown in Figures 7-10 for $\widehat{m}=16,32,64,128$. The elliptical cylinder backscattered RCS using (23) is also shown in Figure 11. Table 2

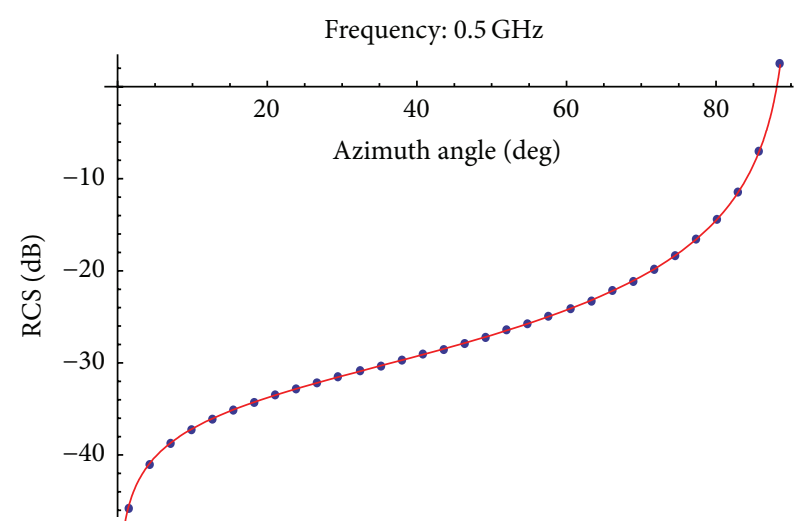

- The reproduced RCS
- The original RCS

FIGURE 3: Backscattered RCS for an elliptical cylinder of $k=5$ and $\varphi=45^{\circ}$.

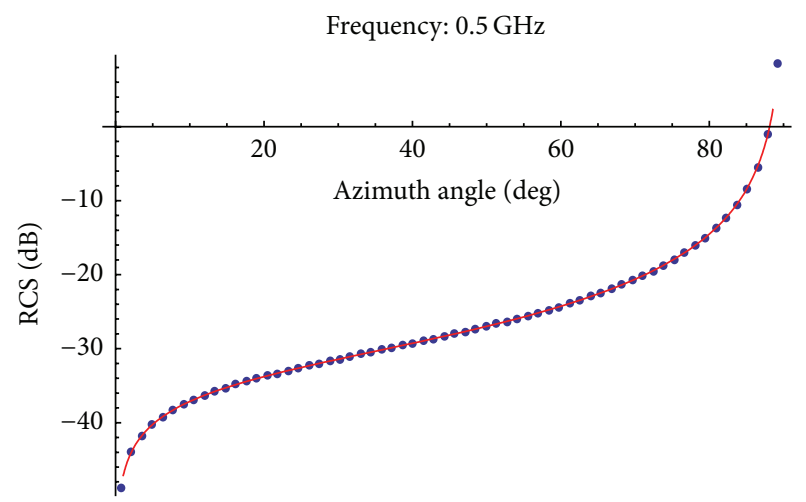

- The reproduced RCS

— The original RCS

FIGURE 4: Backscattered RCS for an elliptical cylinder of $k=6$ and $\varphi=45^{\circ}$.

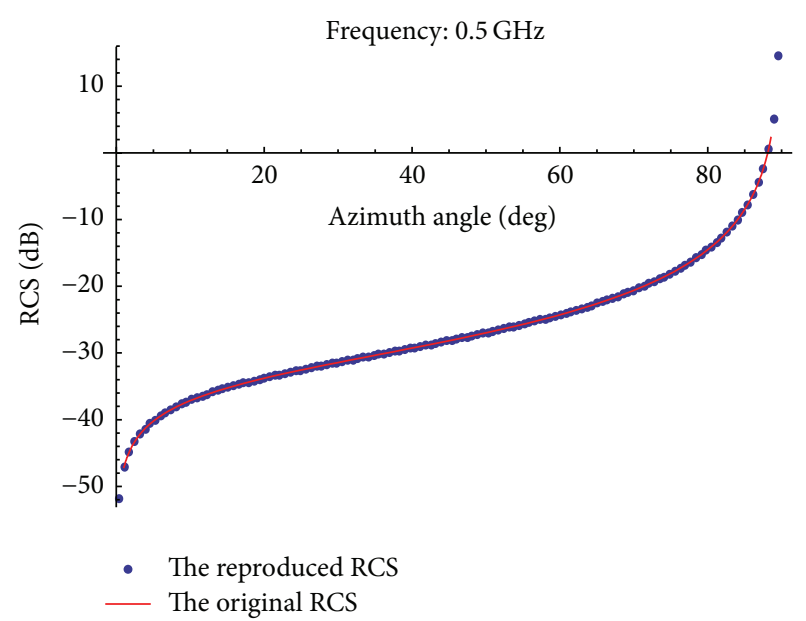

FIgURE 5: Backscattered RCS for an elliptical cylinder of $k=7$ and $\varphi=45^{\circ}$. 


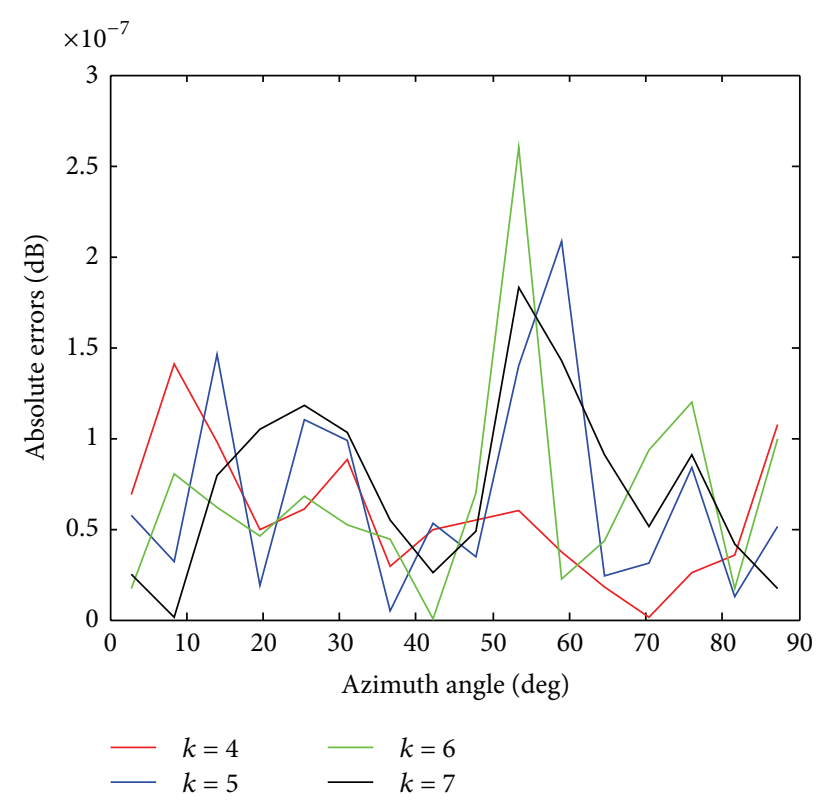

Figure 6: Absolute errors for different $k$ and $\varphi=45^{\circ}$.

TABLE 1: The absolute errors between original RCS and reproduced RCS for different $k(\mathrm{~dB})$.

\begin{tabular}{lcccc}
\hline$\theta \times 90^{\circ}$ & $k=4$ & $k=5$ & $k=6$ & $k=7$ \\
\hline 0.1 & 0.00874 & 0.00540 & 0.00079 & 0.00035 \\
0.2 & 0.02334 & 0.00354 & 0.00157 & 0.00019 \\
0.3 & 0.03044 & 0.00348 & 0.00177 & 0.00024 \\
0.4 & 0.02269 & 0.00924 & 0.00112 & 0.00055 \\
0.5 & 0.25790 & 0.05183 & 0.01178 & 0.00281 \\
0.6 & 0.03000 & 0.01726 & 0.00251 & 0.00113 \\
0.7 & 0.11124 & 0.01937 & 0.00794 & 0.00097 \\
0.8 & 0.32609 & 0.02837 & 0.01630 & 0.00237 \\
0.9 & 6.49094 & 0.32208 & 0.02801 & 0.01609 \\
\hline
\end{tabular}

shows the absolute errors between the original RCS and the reproduced RCS for different values of $\theta, \varphi$, and $\widehat{m}$.

We can see that the reproduced RCS is more and more close to the original RCS with the value of $\widehat{m}$ becoming large by taking a closer look at Figures 7-11 and Table 2.

The second example used to demonstrate this new LWMBPE procedure was a triangular flat defined by the isosceles triangle as oriented in Figure $12(a=0.2 \mathrm{~m}, b=0.75 \mathrm{~m})$. The RCS interpolation used the rational function with a numerator order $n=8$ and a denominator order $n=7$. The frequency domain sampling was done at sixteen frequencies (i.e., $0.5,0.6, \ldots, 1.9$ and $2 \mathrm{GHz}$ ). The backscattered RCS can

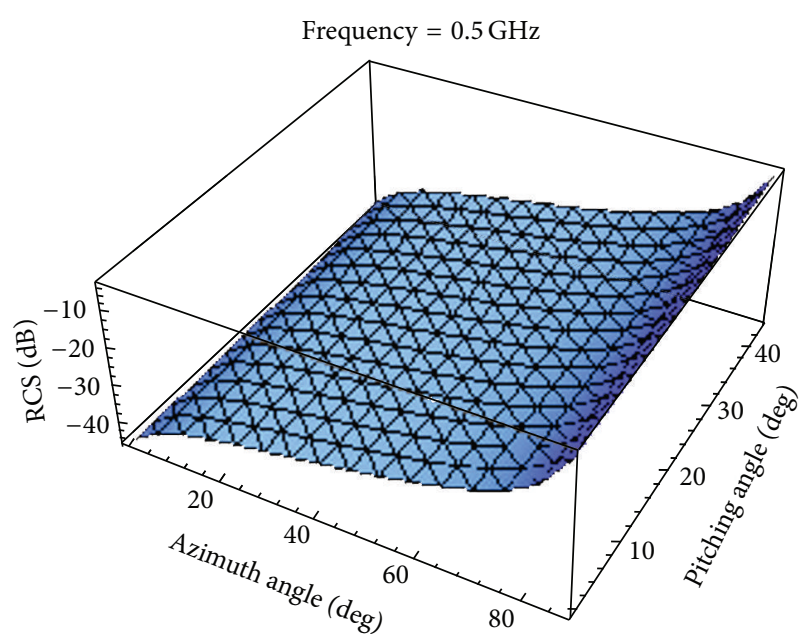

FIGURE 7: Backscattered RCS for an elliptical cylinder for $\theta, \varphi$ of $\widehat{m}=$ 16.

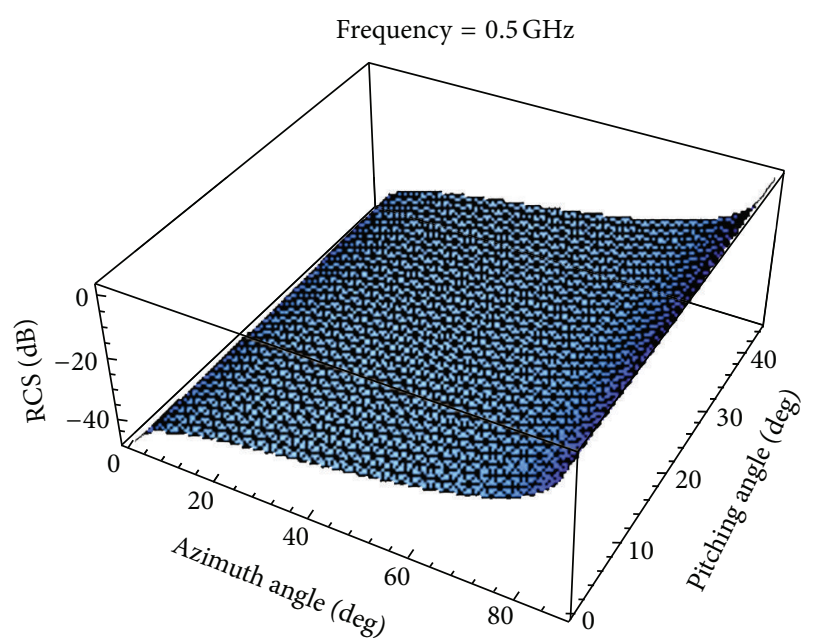

FIGURE 8: Backscattered RCS for an elliptical cylinder for $\theta, \varphi$ of $\widehat{m}=$ 32.

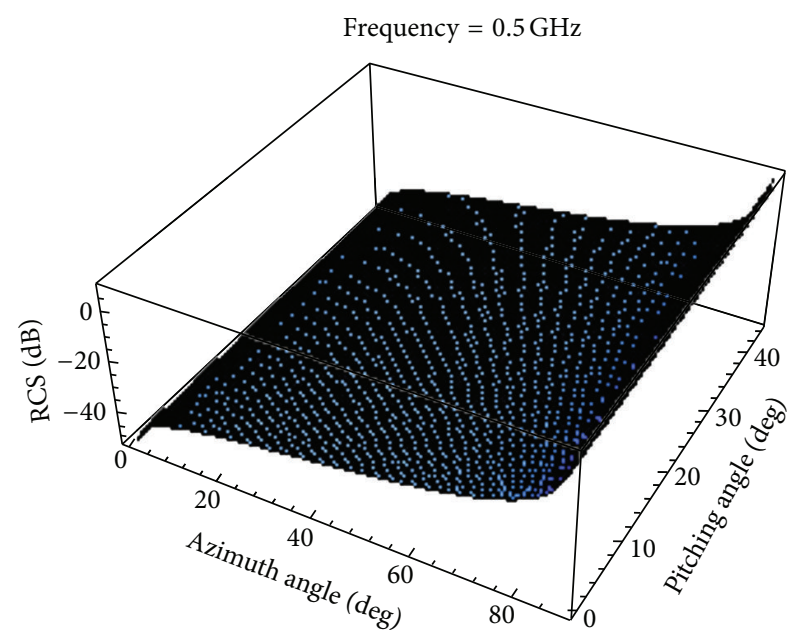

FIGURE 9: Backscattered RCS for an elliptical cylinder for $\theta, \varphi$ of $\widehat{m}=$ 64. 


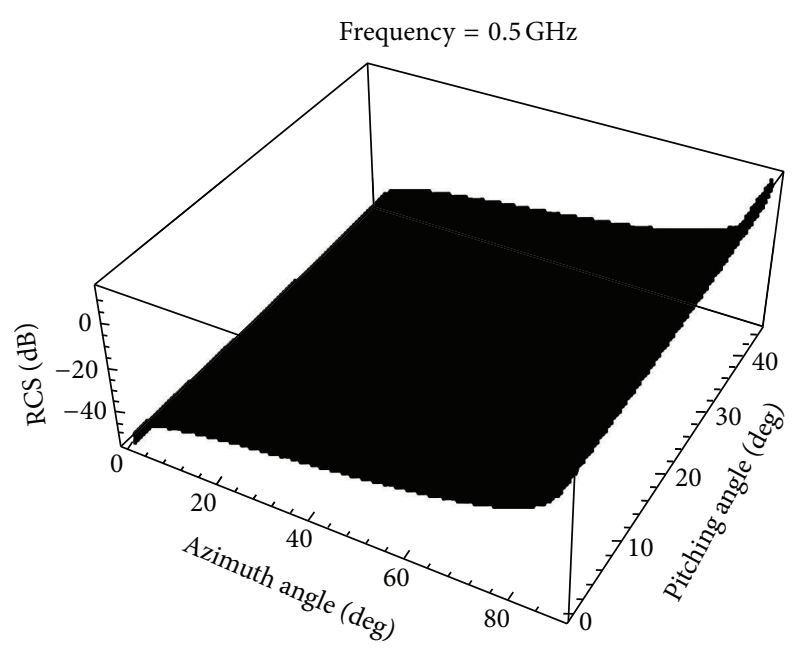

Figure 10: Backscattered RCS for an elliptical cylinder for $\theta, \varphi$ of $\widehat{m}=128$.

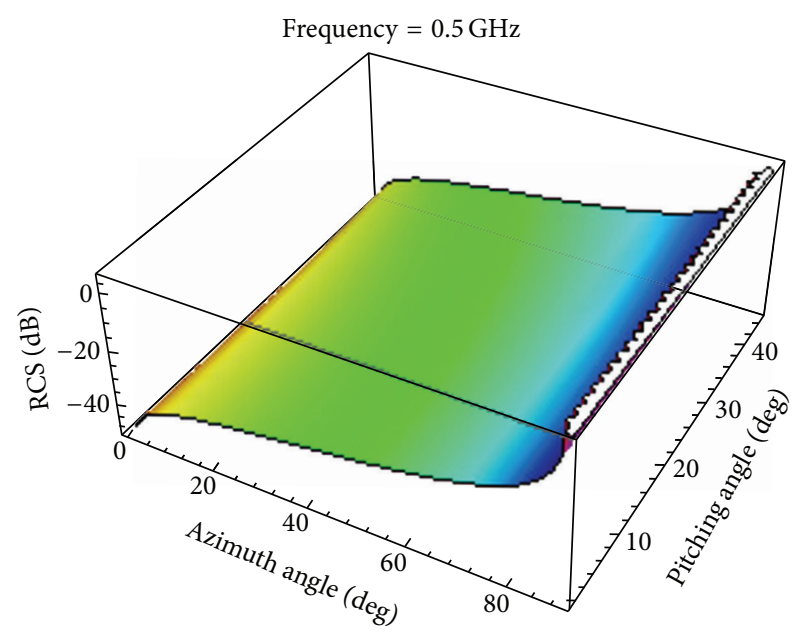

FIGURE 11: The elliptical cylinder backscattered $\operatorname{RCS}$ for $\theta, \varphi$.

be approximated for small aspect angles (less than $30^{\circ}$ ) by [20]

$$
\begin{aligned}
\sigma & =\frac{4 \pi A^{2}}{\lambda^{2}}(\cos \theta)^{2} \sigma_{0} \\
\sigma_{0} & =\frac{\left[(\sin \alpha)^{2}-(\sin (\beta / 2))^{2}\right]^{2}+\sigma_{01}}{\alpha^{2}-(\beta / 2)^{2}}, \\
\sigma_{01} & \\
& =0.25(\sin \varphi)^{2}\left[\left(\frac{2 a}{b}\right) \cos \varphi \sin \beta-\sin \varphi \sin 2 \alpha\right]^{2},
\end{aligned}
$$

where $\alpha=k a \sin \theta \cos \varphi, \beta=k b \sin \theta \sin \varphi$, and $A=a b / 2$.

Based on a perfectly conducting isosceles triangular flat plate, Figures 13, 14, 15, and 16 show the normalized backscattered RCS using (26) and the reproduced RCS using LW-MBPE for $k=4,5,6,7$. The absolute errors for the
TABLE 2: The absolute errors between original RCS and reproduced RCS for different $\widehat{m}(\mathrm{~dB})$.

\begin{tabular}{lllll}
\hline$\left(\theta \times 90^{\circ}, \varphi \times 45^{\circ}\right)$ & $\widehat{m}=16$ & $\widehat{m}=32$ & $\widehat{m}=64$ & $\widehat{m}=128$ \\
\hline$(0.1,0.1)$ & 0.01014 & 0.00610 & 0.00084 & 0.00041 \\
$(0.2,0.2)$ & 0.02629 & 0.00398 & 0.00176 & 0.00021 \\
$(0.3,0.3)$ & 0.03376 & 0.00383 & 0.00208 & 0.00031 \\
$(0.4,0.4)$ & 0.02468 & 0.01020 & 0.00132 & 0.00059 \\
$(0.5,0.5)$ & 0.27407 & 0.05570 & 0.01286 & 0.00302 \\
$(0.6,0.6)$ & 0.03233 & 0.01845 & 0.00270 & 0.00122 \\
$(0.7,0.7)$ & 0.11657 & 0.01995 & 0.00828 & 0.00102 \\
$(0.8,0.8)$ & 0.33268 & 0.02912 & 0.01669 & 0.00241 \\
$(0.9,0.9)$ & 6.49592 & 0.32396 & 0.02837 & 0.01616 \\
\hline
\end{tabular}

TABLE 3: The absolute errors between reproduced RCS and original RCS for different $k(\mathrm{~dB})$.

\begin{tabular}{lcccc}
\hline$\theta \times 90^{\circ}$ & $k=4$ & $k=5$ & $k=6$ & $k=7$ \\
\hline 0.1 & 8.54737 & 5.98691 & 0.86121 & 0.56261 \\
0.2 & 6.56903 & 1.79307 & 1.08659 & 0.14338 \\
0.3 & 3.55198 & 0.15870 & 0.04182 & 0.01021 \\
0.4 & 0.69205 & 0.03673 & 0.03452 & 0.00126 \\
0.5 & 0.33927 & 0.10280 & 0.02992 & 0.01416 \\
0.6 & 2.76694 & 0.71435 & 0.21953 & 0.00813 \\
0.7 & 0.61607 & 0.03730 & 0.01911 & 0.00141 \\
0.8 & 0.04907 & 0.01372 & 0.00938 & 0.00182 \\
0.9 & 0.09652 & 0.07825 & 0.05342 & 0.04251 \\
\hline
\end{tabular}

reproduced RCS and original RCS in Figures 13-16 are shown in Figure 17.

Table 3 shows the absolute errors between the original RCS and the reproduced RCS. From Table 3, we can also find that the errors are smaller and smaller with $k$ increasing.

The interpolated RCS as a function of azimuth angle $\theta$ and frequency is shown in Figures 18-21 for different $\widehat{m}$.

According to the above analysis, we can acquire the approximate backscattered RCS of the perfectly conducting triangular flat plate for arbitrary $\theta$ and frequency by using LW-MBPE method. What is more, the approximations are more and more accurate with $\widehat{m}$ increasing. It is evident from the examples that the LW-MBPE method is convergent.

In the two examples, the approximate formulas of the target backscattered RCS are known. However, in practical, the majority of targets backscattered RCS are unknown, and approximate formulas cannot be found. We can also obtain the value of RCS of sampling points by using the method of moments (MoM). 


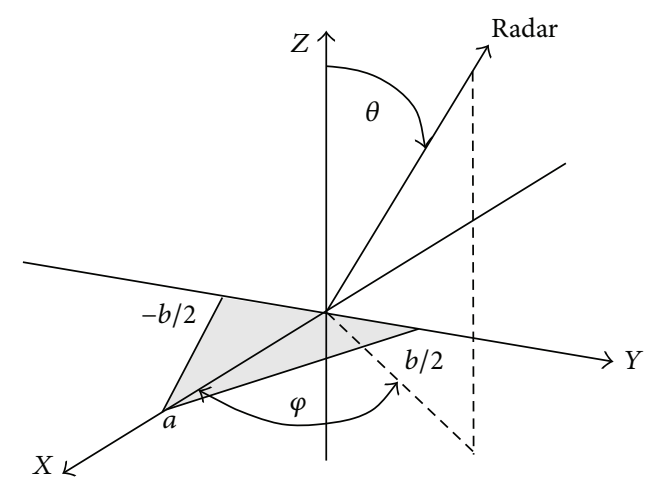

FIGURE 12: Coordinates for a perfectly conducting isosceles triangular flat plate.

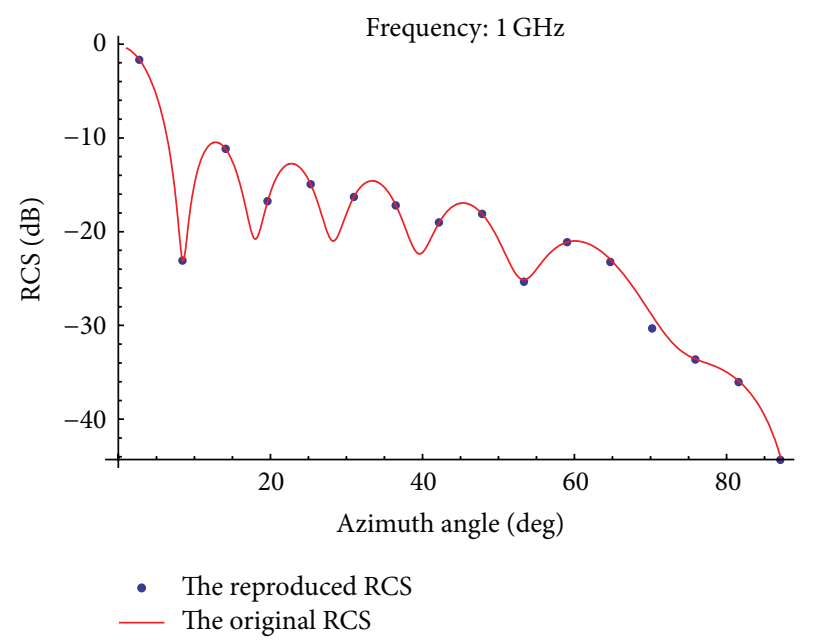

FIGURE 13: Backscattered RCS for a perfectly conducting triangular flat plate, $k=4$ and $\varphi=60^{\circ}$.

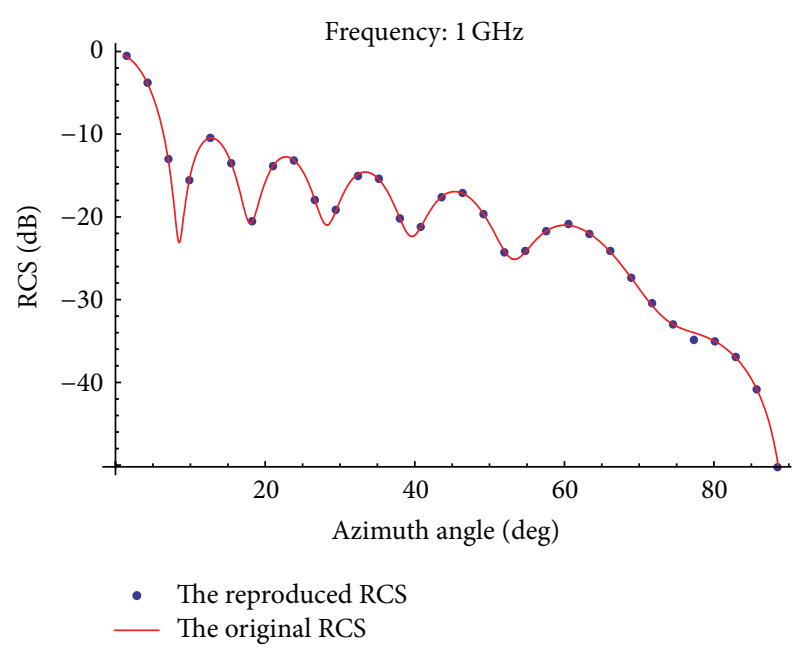

FIGURE 14: Backscattered RCS for a perfectly conducting triangular flat plate, $k=5$ and $\varphi=60^{\circ}$.

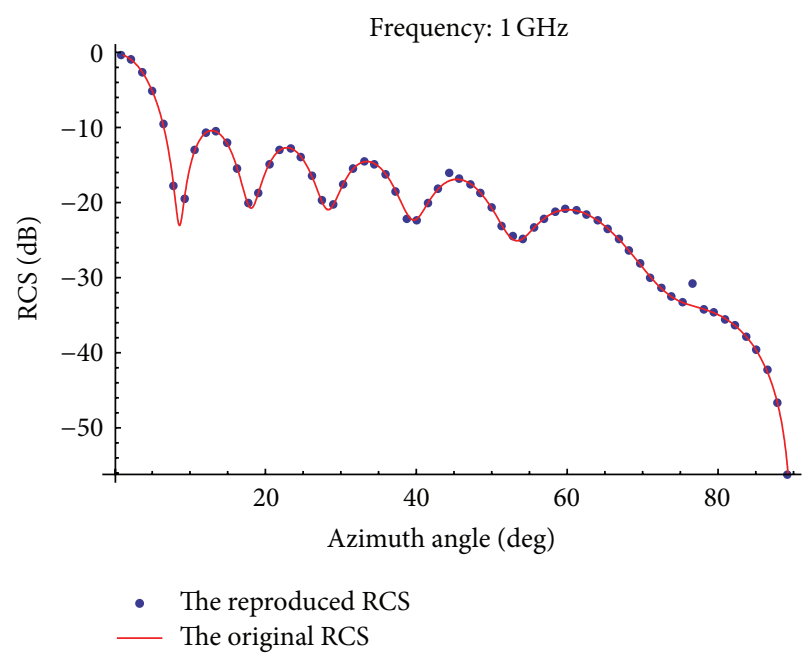

FIGURE 15: Backscattered RCS for a perfectly conducting triangular flat plate, $k=6$ and $\varphi=60^{\circ}$.

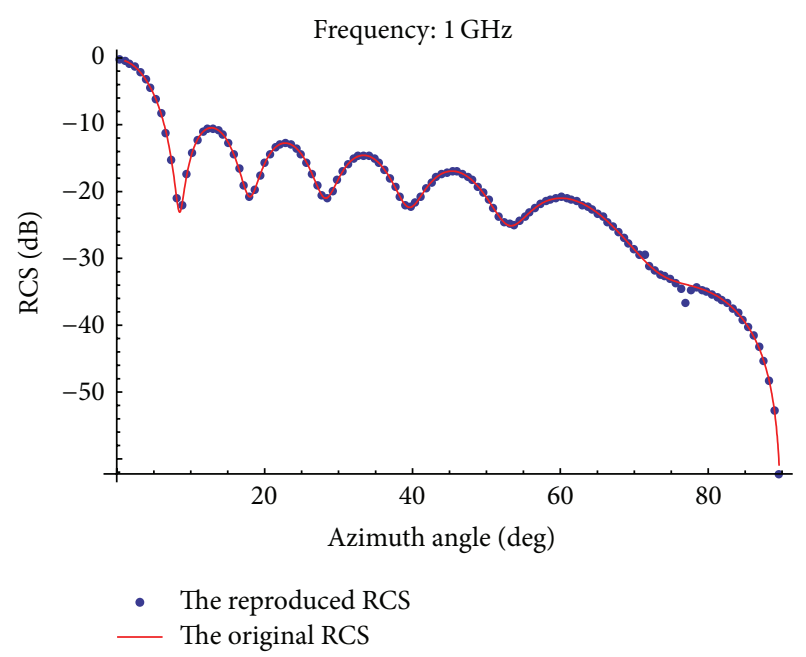

FIGURE 16: Backscattered RCS for a perfectly conducting triangular flat plate, $k=7$ and $\varphi=60^{\circ}$.

\section{Conclusion}

In this paper, a scheme to interpolate target RCS in both the frequency and spatial domains simultaneously using LWMBPE method was proposed. The Padé rational function fitting model used for MBPE in the frequency domain can be easily modified to include spatial dependence on its numerator and denominator coefficients. This interpolation technique was applied to two examples, an elliptical cylinder and a perfectly conducting triangular flat plate; in each case, the modified Padé rational function yielded excellent coincidence with the exact results calculated using their RCS formulas. The results also show that the proposed method is convergent with sampling points increasing. 


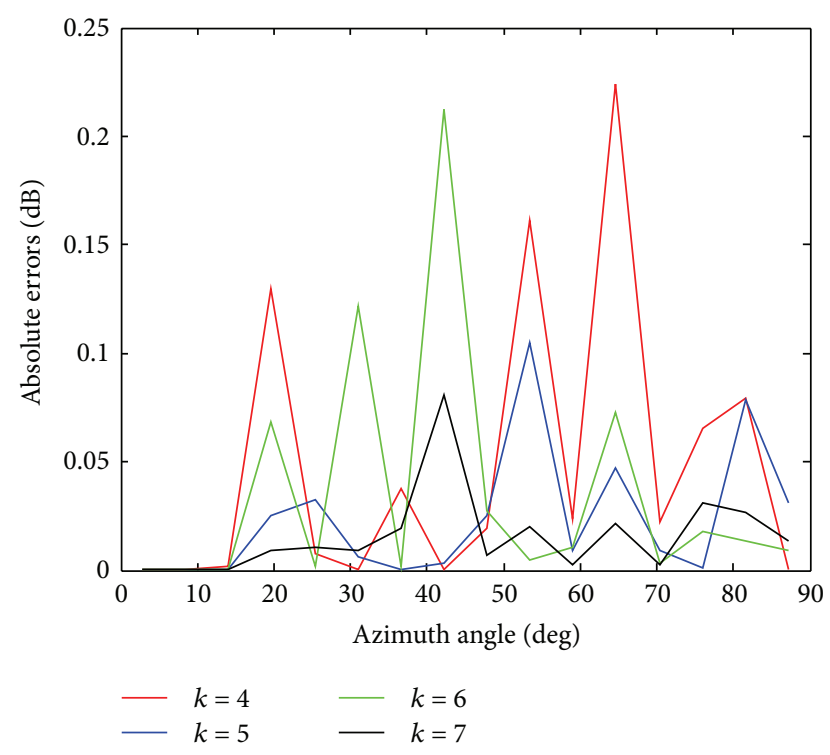

FIGURE 17: Absolute errors for different $k$ and $\varphi=60^{\circ}$.

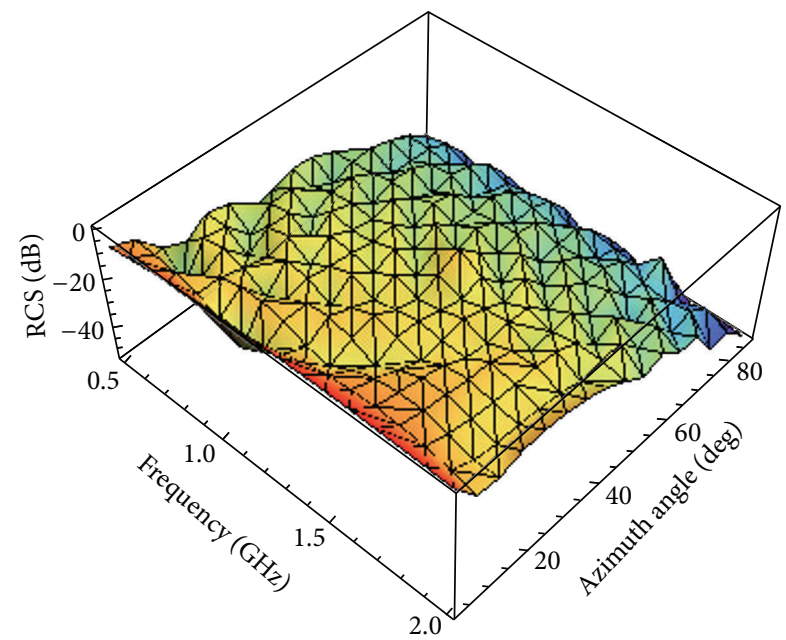

FIGURE 18: Backscattered RCS for a perfectly conducting triangular flat plate for $\theta$ and frequency of $\widehat{m}=16$.

\section{Appendices}

\section{A. The proof of Theorem 1}

Proof. Let $F\left(\theta^{\prime}, s_{l}\right)$ be a function defined on $[0,1]$ such that

$$
\left|F^{\prime \prime}\left(\theta^{\prime}, s_{l}\right)\right| \leq \widetilde{M},
$$

where $\widetilde{M}$ is a positive constant.

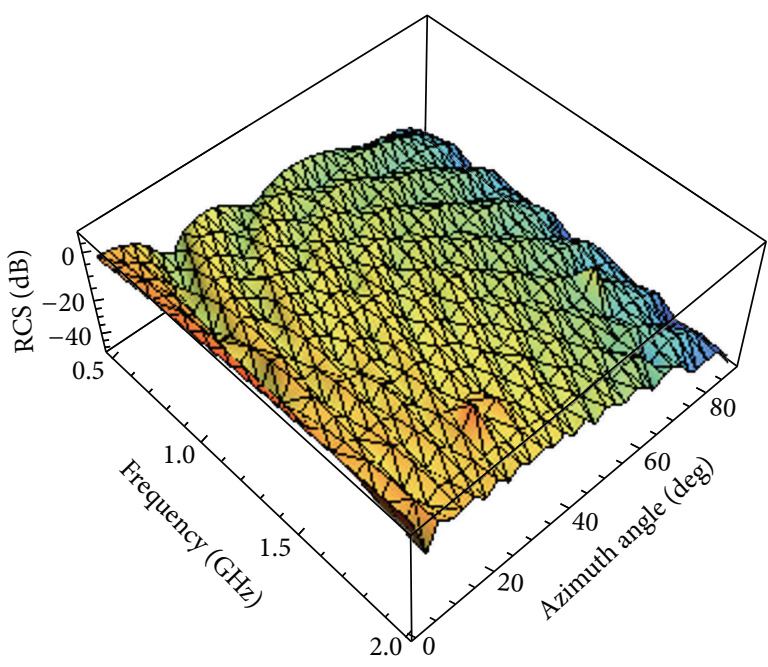

FIGURE 19: Backscattered RCS for a perfectly conducting triangular flat plate for $\theta$ and frequency of $\widehat{m}=32$.

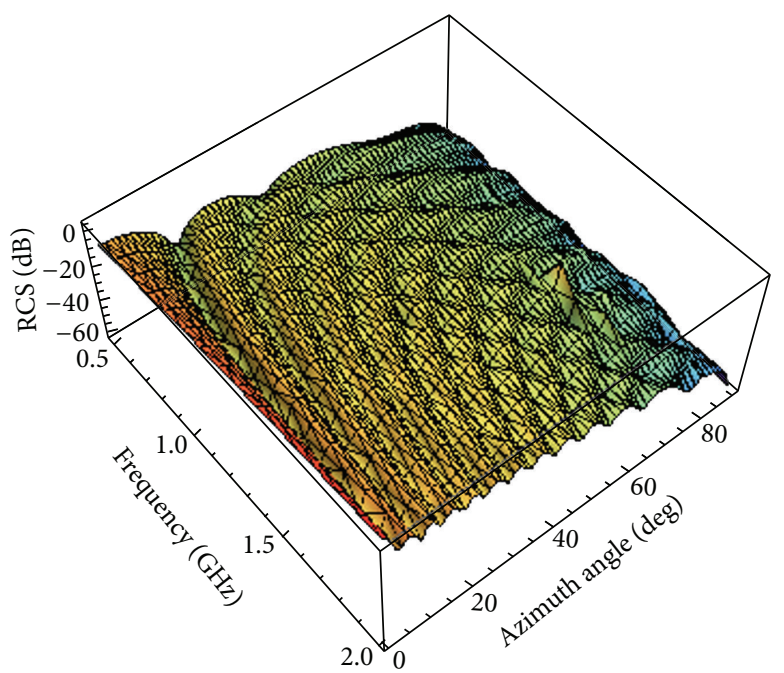

FIGURE 20: Backscattered RCS for a perfectly conducting triangular flat plate for $\theta$ and frequency of $\widehat{m}=64$.

The orthonormality of sequence $\left\{\psi_{n m}\left(\theta^{\prime}\right)\right\}$ on $[0,1)$ implies that $\int_{0}^{1} \Psi\left(\theta^{\prime}\right)\left[\Psi\left(\theta^{\prime}\right)\right]^{T} d \theta^{\prime}=I$, where $I$ is the identity matrix; then

$$
\begin{aligned}
\| F & \left(\theta^{\prime}, s_{l}\right)-F_{k, M}\left(\theta^{\prime}, s_{l}\right) \|_{E}^{2} \\
& =\int_{0}^{1}\left(F\left(\theta^{\prime}, s_{l}\right)-F_{k, M}\left(\theta^{\prime}, s_{l}\right)\right)^{2} d \theta^{\prime} \\
& =\int_{0}^{1}\left(\sum_{n=2^{k}}^{+\infty} \sum_{m=M}^{+\infty} c_{n m} \psi_{n m}\left(\theta^{\prime}\right)\right)^{2} d \theta^{\prime} \\
& =\sum_{n=2^{k}}^{+\infty} \sum_{m=M}^{+\infty} c_{n m}^{2} \int_{0}^{1} \psi_{n m}^{2}\left(\theta^{\prime}\right) d \theta^{\prime}=\sum_{n=2^{k}}^{+\infty} \sum_{m=M}^{+\infty} c_{n m}^{2},
\end{aligned}
$$




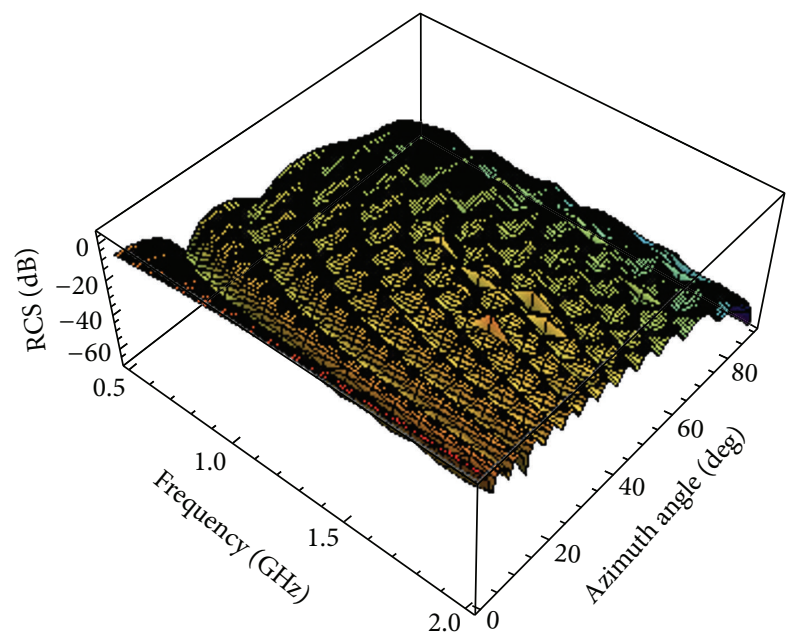

FIGURE 21: Backscattered RCS for a perfectly conducting triangular flat plate for $\theta$ and frequency of $\widehat{m}=128$.

where $c_{n m}=\left\langle F\left(\theta^{\prime}, s_{l}\right), \psi_{n m}\left(\theta^{\prime}\right)\right\rangle=\int_{0}^{1} F\left(\theta^{\prime}, s_{l}\right) \cdot \psi_{n m}\left(\theta^{\prime}\right) d \theta^{\prime}$; then we can get

$$
\begin{gathered}
c_{n m}=\int_{0}^{1} F\left(\theta^{\prime}, s_{l}\right) \psi_{n m}\left(\theta^{\prime}\right) d \theta^{\prime}=\int_{(\widehat{n}-1) / 2^{k}}^{(\hat{n}+1) / 2^{k}} F\left(\theta^{\prime}, s_{l}\right) \\
\cdot\left(\frac{2 m+1}{2}\right)^{1 / 2} 2^{k / 2} P_{m}\left(2^{k} \theta^{\prime}-\widehat{n}\right) d \theta^{\prime} .
\end{gathered}
$$

Now, let $2^{k} \theta^{\prime}-\widehat{n}=t$; then

$$
\begin{aligned}
c_{n m} & =\int_{-1}^{1} F\left(\frac{\widehat{n}+t}{2^{k}}, s_{l}\right)\left(\frac{2 m+1}{2}\right)^{1 / 2} 2^{k / 2} P_{m}(t) \frac{1}{2^{k}} d t \\
& =-\left(\frac{1}{2^{3 k+1}(2 m+1)}\right)^{1 / 2} \int_{-1}^{1} F^{\prime}\left(\frac{\widehat{n}+t}{2^{k}}, s_{l}\right)\left(P_{m+1}(t)\right. \\
& \left.-P_{m-1}(t)\right) d t=-\left(\frac{1}{2^{3 k+1}(2 m+1)}\right)^{1 / 2} \int_{-1}^{1} F^{\prime}\left(\frac{\widehat{n}+t}{2^{k}}\right. \\
& \left.s_{l}\right) d\left(\frac{P_{m+2}(t)-P_{m}(t)}{2 m+3}-\frac{P_{m}(t)-P_{m-2}(t)}{2 m-1}\right) \\
& =\left(\frac{1}{2^{5 k+1}(2 m+1)}\right)^{1 / 2} \int_{-1}^{1} F^{\prime \prime}\left(\frac{\widehat{n}+t}{2^{k}}, s_{l}\right) \\
& \cdot\left(\frac{P_{m+2}(t)-P_{m}(t)}{2 m+3}-\frac{P_{m}(t)-P_{m-2}(t)}{2 m-1}\right) d t .
\end{aligned}
$$

$$
\begin{aligned}
\left|c_{n m}\right|^{2} & =\left|\left(\frac{1}{2^{5 k+1}(2 m+1)}\right)^{1 / 2} \int_{-1}^{1} F^{\prime \prime}\left(\frac{\widehat{n}+t}{2^{k}}, s_{l}\right)\left(\frac{P_{m+2}(t)-P_{m}(t)}{2 m+3}-\frac{P_{m}(t)-P_{m-2}(t)}{2 m-1}\right) d t\right|^{2} \\
& =\frac{1}{2^{5 k+1}(2 m+1)}\left|\int_{-1}^{1} F^{\prime \prime}\left(\frac{\widehat{n}+t}{2^{k}}, s_{l}\right) \frac{(2 m-1) P_{m+2}(t)-(4 m+2) P_{m}(t)+(2 m+3) P_{m-2}(t)}{(2 m+3)(2 m-1)} d t\right|^{2} \\
& \leq \frac{1}{2^{5 k+1}(2 m+1)} \int_{-1}^{1}\left|F^{\prime \prime}\left(\frac{\widehat{n}+t}{2^{k}}, s_{l}\right)\right|^{2} d t \int_{-1}^{1}\left|\frac{(2 m-1) P_{m+2}(t)-(4 m+2) P_{m}(t)+(2 m+3) P_{m-2}(t)}{(2 m+3)(2 m-1)}\right|^{2} d t \\
& <\frac{12 \widetilde{M}^{2}}{(2 n)^{5}(2 m-3)^{4}} .
\end{aligned}
$$

Therefore, we have

$$
\begin{aligned}
\sum_{n=2^{k}}^{+\infty} \sum_{m=M}^{+\infty} c_{n m}^{2} & <\sum_{n=2^{k}}^{+\infty} \sum_{m=M}^{+\infty} \frac{12 \widetilde{M}^{2}}{(2 n)^{5}(2 m-3)^{4}} \\
& =12 \widetilde{M}^{2} \sum_{n=2^{k}}^{+\infty} \frac{1}{(2 n)^{5}} \sum_{m=M}^{+\infty} \frac{1}{(2 m-3)^{4}} \\
& =\frac{\widetilde{M}^{2}}{31} \frac{1}{2^{5 k+3}}\left(\frac{\Gamma^{\prime}(M-1.5)}{\Gamma(M-1.5)}\right)^{\prime \prime \prime} .
\end{aligned}
$$

Then we obtain

$$
\begin{aligned}
& \left\|F\left(\theta^{\prime}, s_{l}\right)-F_{k, M}\left(\theta^{\prime}, s_{l}\right)\right\|_{E}^{2} \\
& \quad \leq \frac{\widetilde{M}^{2}}{31} \frac{1}{2^{5 k+3}}\left(\frac{\Gamma^{\prime}(M-1.5)}{\Gamma(M-1.5)}\right)^{\prime \prime \prime} ;
\end{aligned}
$$

namely,

$$
\begin{aligned}
& \left\|F\left(\theta^{\prime}, s_{l}\right)-F_{k, M}\left(\theta^{\prime}, s_{l}\right)\right\|_{E} \\
& \quad \leq\left(\frac{\widetilde{M}^{2}}{31} \frac{1}{2^{5 k+3}}\left(\frac{\Gamma^{\prime}(M-1.5)}{\Gamma(M-1.5)}\right)^{\prime \prime \prime}\right)^{1 / 2} .
\end{aligned}
$$

This completes the proof. 


\section{B. The proof of Theorem 2}

Proof. The orthonormality of the sequence $\left\{\psi_{i}(\theta)\right\}$ on $[0,1)$ implies that $\int_{0}^{1} \Psi(\theta)[\Psi(\theta)]^{T} d x=I$, where $I$ is the identity matrix; then

$$
\begin{aligned}
& \left\|F\left(\theta, \varphi, s_{l}\right)-F_{\widehat{m}}\left(\theta, \varphi, s_{l}\right)\right\|_{E}^{2} \\
& =\sum_{i=\widehat{m}+1}^{\infty} \sum_{j=\widehat{m}+1}^{\infty} \sum_{i^{\prime}=\widehat{m}+1}^{\infty} \sum_{j^{\prime}=\widehat{m}+1}^{\infty} u_{i j} u_{i^{\prime} j^{\prime}} \\
& \cdot \int_{0}^{1} \int_{0}^{1} \psi_{i}(\theta) \psi_{j}(\varphi) d \theta d \varphi \\
& \cdot \int_{0}^{1} \int_{0}^{1} \psi_{i^{\prime}}(\theta) \psi_{j^{\prime}}(\varphi) d \theta d \varphi=\sum_{i=\widehat{m}+1}^{\infty} \sum_{j=\widehat{m}+1}^{\infty} u_{i j}^{2} .
\end{aligned}
$$

The Legendre wavelets coefficients of function $F\left(\theta, \varphi, s_{l}\right)$ are defined by

$$
\begin{aligned}
u_{i j} & =\int_{0}^{1} \int_{0}^{1} F\left(\theta, \varphi, s_{l}\right) \psi_{i}(\theta) \psi_{j}(\varphi) d \theta d \varphi \\
& =\int_{0}^{1} \int_{I_{n k}} F\left(\theta, \varphi, s_{l}\right)\left(\frac{2 m+1}{2}\right)^{1 / 2} \\
& \cdot 2^{k / 2} P_{m}\left(2^{k} \theta-\widehat{n}\right) \psi_{j}(\varphi) d \theta d \varphi .
\end{aligned}
$$

Let $2^{k} \theta-\widehat{n}=t$; by change of $2^{k} \theta-\widehat{n}=t$ and $d \theta=\left(1 / 2^{k}\right) d t$, we obtain

$$
\begin{aligned}
u_{i j} & =\left(\frac{2 m+1}{2}\right)^{1 / 2} 2^{-k / 2} \int_{0}^{1} \psi_{j}(\varphi) \int_{-1}^{1} F\left(\frac{\widehat{n}+t}{2^{k}}, \varphi, s_{l}\right) P_{m}(t) d t d \varphi \\
& =\left(\frac{1}{2^{k+1}(2 m+1)}\right)^{1 / 2} \int_{0}^{1} \psi_{j}(\varphi) \int_{-1}^{1} F\left(\frac{\widehat{n}+t}{2^{k}}, \varphi,\right. \\
& \left.s_{l}\right) d\left(P_{m+1}(t)-P_{m-1}(t)\right) d \varphi \\
& =-\left(\frac{1}{2^{3 k+1}(2 m+1)}\right)^{1 / 2} \int_{0}^{1} \psi_{j}(\varphi) \int_{-1}^{1} \frac{\partial}{\partial t} F\left(\frac{\widehat{n}+t}{2^{k}}, \varphi,\right. \\
& \left.s_{l}\right) d\left(\frac{P_{m+2}(t)-P_{m}(t)}{2 m+3}-\frac{P_{m}(t)-P_{m-2}(t)}{2 m-1}\right) d \varphi \\
& =\left(\frac{1}{2^{5 k+1}(2 m+1)}\right)^{1 / 2} \int_{0}^{1} \psi_{j}(\varphi) \int_{-1}^{1} \frac{\partial^{2}}{\partial t^{2}} F\left(\frac{\widehat{n}+t}{2^{k}}, \varphi, s_{l}\right) \\
& \cdot\left(\frac{P_{m+2}(t)-P_{m}(t)}{2 m+3}-\frac{P_{m}(t)-P_{m-2}(t)}{2 m-1}\right) d t d \varphi .
\end{aligned}
$$

Now, let $\tau_{m}(t)=(2 m-1) P_{m+2}(t)-2(2 m+1) P_{m}(t)+(2 m+$ 3) $P_{m-2}(t)$; then we have

$$
\begin{aligned}
u_{i j} & =\left(\frac{1}{2^{5 k+1}(2 m+1)}\right)^{1 / 2} \frac{1}{(2 m-1)(2 m+3)} \\
& \cdot \int_{0}^{1} \psi_{j}(\varphi) \int_{-1}^{1} \frac{\partial^{2}}{\partial t^{2}} F\left(\frac{\widehat{n}+t}{2^{k}}, \varphi, s_{l}\right) \tau_{m}(t) d t d \varphi .
\end{aligned}
$$

By solving this equation, we have

$$
\begin{aligned}
& u_{i j}=A(k, m) \int_{-1}^{1} \int_{-1}^{1} \frac{\partial^{4}}{\partial t^{2} \partial s^{2}} F\left(\frac{\widehat{n}+t}{2^{k}}, \frac{\widehat{n}+s}{2^{k}}, s_{l}\right) \\
& \cdot \tau_{m}(t) \tau_{m}(s) d t d s,
\end{aligned}
$$

where $A(k, m)=\left(1 / 2^{5 k+1}(2 m+1)\right)\left(1 /(2 m-1)^{2}(2 m+3)^{2}\right)$.

So we have

$$
\begin{aligned}
& \left|u_{i j}\right| \leq A(k, m) \int_{-1}^{1} \int_{-1}^{1}\left|\frac{\partial^{4}}{\partial t^{2} \partial s^{2}} F\left(\frac{\widehat{n}+t}{2^{k}}, \frac{\widehat{n}+s}{2^{k}}, s_{l}\right)\right| \\
& \cdot\left|\tau_{m}(t)\right|\left|\tau_{m}(s)\right| d t d s .
\end{aligned}
$$

Moreover, it was shown in the above equation that

$$
\int_{-1}^{1}\left|\tau_{m}(t)\right| d t \leq \sqrt{24} \frac{2 m+3}{\sqrt{2 m-3}}
$$

thus, we have

$$
\begin{aligned}
\left|u_{i j}\right| & \leq A(k, m) \frac{24 \widehat{M}(2 m+3)^{2}}{2 m-3} \\
& \leq \frac{1}{2^{5 k}(2 m+1)} \frac{1}{(2 m-3)(2 m-1)^{2}} \\
& \leq \frac{12 \widehat{M}}{(2 n)^{5}(2 m-3)^{4}} .
\end{aligned}
$$

Namely,

$$
\left|u_{i j}\right|^{2} \leq \frac{144 \widehat{M}^{2}}{(2 n)^{10}(2 m-3)^{8}} .
$$

Therefore, we have

$$
\begin{aligned}
& \left\|F\left(\theta, \varphi, s_{l}\right)-F_{\widehat{m}}\left(\theta, \varphi, s_{l}\right)\right\|_{E}^{2} \leq \sum_{i=\widehat{m}}^{\infty} \sum_{j=\widehat{m}}^{\infty} u_{i j}^{2} \\
& \leq \sum_{i=\widehat{m}}^{\infty} \sum_{j=\widehat{m}}^{\infty} \frac{144 \widehat{M}^{2}}{(2 n)^{10}(2 m-3)^{8}} \\
& \leq \sum_{M=1}^{\infty} \sum_{p=k-1}^{\infty}\left(\sum_{i=M 2^{p}}^{M 2^{p+1}-1 M 2^{p+1}-1} \sum_{j=M 2^{p}} \frac{144 \widehat{M}^{2}}{\left(2^{k}\right)^{10}(2 M-5)^{8}}\right) \\
& \leq \sum_{M=1}^{\infty} \sum_{p=k-1}^{\infty}\left(\frac{144 \widehat{M}^{2}}{\left(2^{p+1}\right)^{10}(2 M-5)^{8}} M^{2} 2^{2 p}\right) \\
& \leq \frac{\widehat{M}^{2} \widehat{N}}{2^{1+8 k}}
\end{aligned}
$$

where $\widehat{N}$ is a constant.

Then we get

$$
\left\|F\left(\theta, \varphi, s_{l}\right)-F_{\widehat{m}}\left(\theta, \varphi, s_{l}\right)\right\|_{E}^{2} \leq \frac{\widehat{M}^{2} \widehat{N}}{2^{8 k}} ;
$$

thus,

$$
\left\|F\left(\theta, \varphi, s_{l}\right)-F_{\widehat{m}}\left(\theta, \varphi, s_{l}\right)\right\|_{E} \leq \frac{\widehat{M} \cdot \widehat{N}^{1 / 2}}{2^{4 k}} .
$$

This completes the proof. 


\section{Nomenclature}

$\psi_{n m}(x)$ : Legendre wavelets function

$P_{m}(x)$ : Legendre polynomials

$c_{i}, u_{i j}$ : Wavelets coefficients

$F(s)$ : Padé rational function

$N_{i}^{j}, D_{i}^{j}$ : Padé rational function coefficients

$\theta: \quad$ Azimuth angle

$\varphi$ : $\quad$ Pitching angle

$\sigma: \quad$ Radar cross section

$f: \quad$ Frequency

$\widehat{m}: \quad$ Positive integer

$P_{i}$ : $\quad$ Power density, or intensity, of a plane wave striking the target

$P_{s}: \quad$ Power per unit solid angle reflected by the target.

\section{Conflict of Interests}

There is no conflict of interests related to this paper.

\section{Acknowledgment}

This work was supported by the National Natural Science Foundation of China under Grant no. 51307004.

\section{References}

[1] E. B. Robert, The Fundamentals of Aircraft Combat Survivability Analysis and Design, Aviation Industry Press, Beijing, China, 1998.

[2] L. F. Ding and F. L. Geng, Radar Principle, XiDian University Press, Xian, China, 2002.

[3] E. F. Knott, J. F. Shaeffer, and M. T. Tuley, Radar Cross Section, SciTech Publishing, 2004.

[4] D. C. Jenn, Radar and Laser Cross Section Engineering, American Institute of Aeronautics and Astronautics, 2005.

[5] C. W. Qu and Y. C. Xiang, "Active cancellation stealth analysis based on RCS characteristic of target," Radar Science and Technology, vol. 8, no. 2, pp. 109-112, 2010.

[6] C. W. Qu, Y. C. Xiang, H. P. Hou, and W. J. Zhou, "Cancellation interference analysis of coherent radar based on phase modulation," Journal of the University of Electronic Science and Technology of China, vol. 40, no. 6, pp. 829-834, 2011.

[7] Y.-C. Xiang, C.-W. Qu, B.-R. Li, and H.-P. Hou, "Simulation research on cancellation stealth of warship based on its radar scattering properties," Journal of System Simulation, vol. 25, no. 1, pp. 104-110, 2013.

[8] E. K. Miller, "Computing radiation and scattering patterns using model-based parameter estimation," in Proceedings of the IEEE Antennas and Propagation Society International Symposium, vol. 1, pp. 66-69, IEEE, Atlanta, Ga, USA, June 1998.

[9] E. K. Miller, "Using adaptive estimation to minimize the number of samples needed to develop a radiation or scattering pattern to a specified uncertainty," Applied Computational Electromagnetics Society Journal, vol. 17, no. 3, pp. 176-186, 2002.

[10] R. J. Allard and D. H. Werner, "The model-based parameter estimation of antenna radiation patterns using windowed interpolation and spherical harmonics," IEEE Transactions on Antennas and Propagation, vol. 51, no. 8, pp. 1891-1906, 2003.
[11] J. Guo, J. Zou, B. Zhang, J. L. He, and Z. C. Guan, "An interpolation model to accelerate the frequency-domain response calculation of grounding systems using the method of moments," IEEE Transactions on Power Delivery, vol. 21, no. 1, pp. 121-128, 2006.

[12] Y. Xiong, D. G. Fang, and W. X. Sheng, "Simultaneous interpolation of RCS in both the spatial and frequency domains using model-based parameter estimation," Chinese Journal of Radio Science, vol. 16, no. 3, pp. 287-290, 2001.

[13] D. H. Werner and R. J. Allard, "The simultaneous interpolation of antenna radiation patterns in both the spatial and frequency domains using model-based parameter estimation," IEEE Transactions on Antennas and Propagation, vol. 48, no. 3, pp. 383-392, 2000.

[14] E. K. Miller, "Model-based parameter estimation in electromagnetics: part I. Background and theoretical development," Applied Computational Electromagnetics Society Newsletter, vol. 10, no. 3, pp. 40-63, 1995.

[15] G. Beylkin, R. Coifman, and V. Rokhlin, "Fast wavelet transforms and numerical algorithms. I," Communications on Pure and Applied Mathematics, vol. 44, no. 2, pp. 141-183, 1991.

[16] Z. C. Li and J. S. Luo, Wavelet Analysis and Its Application, Electronic Industrial Publication, Beijing, China, 2005.

[17] Z. X. Ge and W. Sha, Wavelet Analysis Theorem and MATLAB Application, Electronic Industrial Publication, Beijing, China, 2007.

[18] H. Jafari, S. A. Yousefi, M. A. Firoozjaee, S. Momani, and C. M. Khalique, "Application of Legendre wavelets for solving fractional differential equations," Computers \& Mathematics with Applications, vol. 62, no. 3, pp. 1038-1045, 2011.

[19] N. Liu and E.-B. Lin, "Legendre wavelet method for numerical solutions of partial differential equations," Numerical Methods for Partial Differential Equations, vol. 26, no. 1, pp. 81-94, 2010.

[20] B. R. Mahafza, Radar Systems Analysis and Design Using MATLAB, Taylor \& Francis, 2nd edition, 2005. 

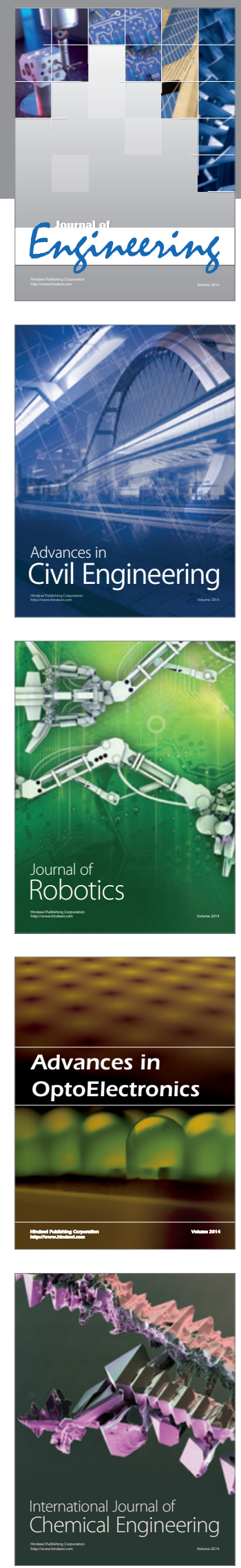

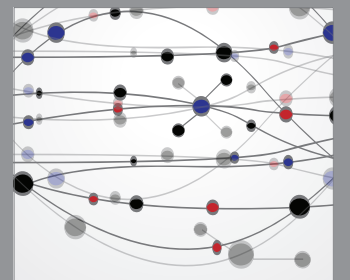

The Scientific World Journal
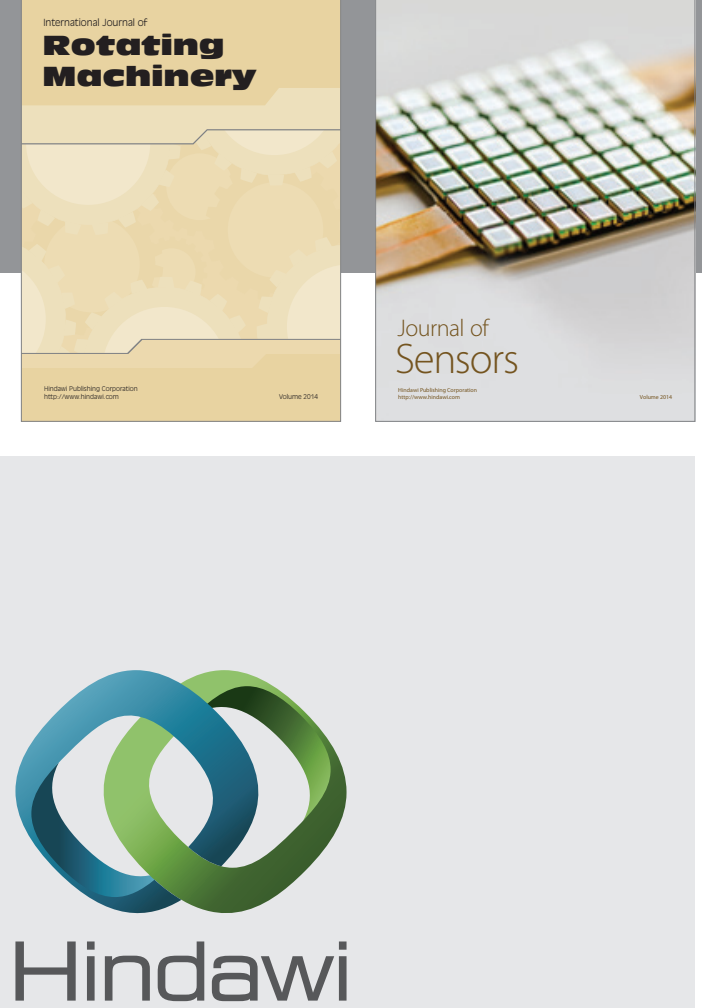

Submit your manuscripts at http://www.hindawi.com
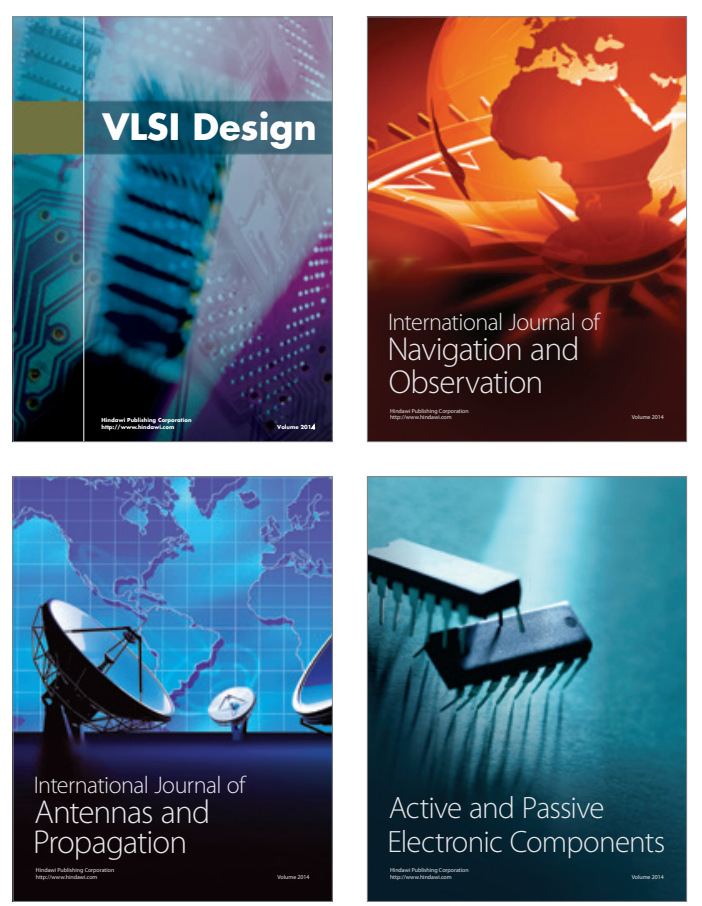
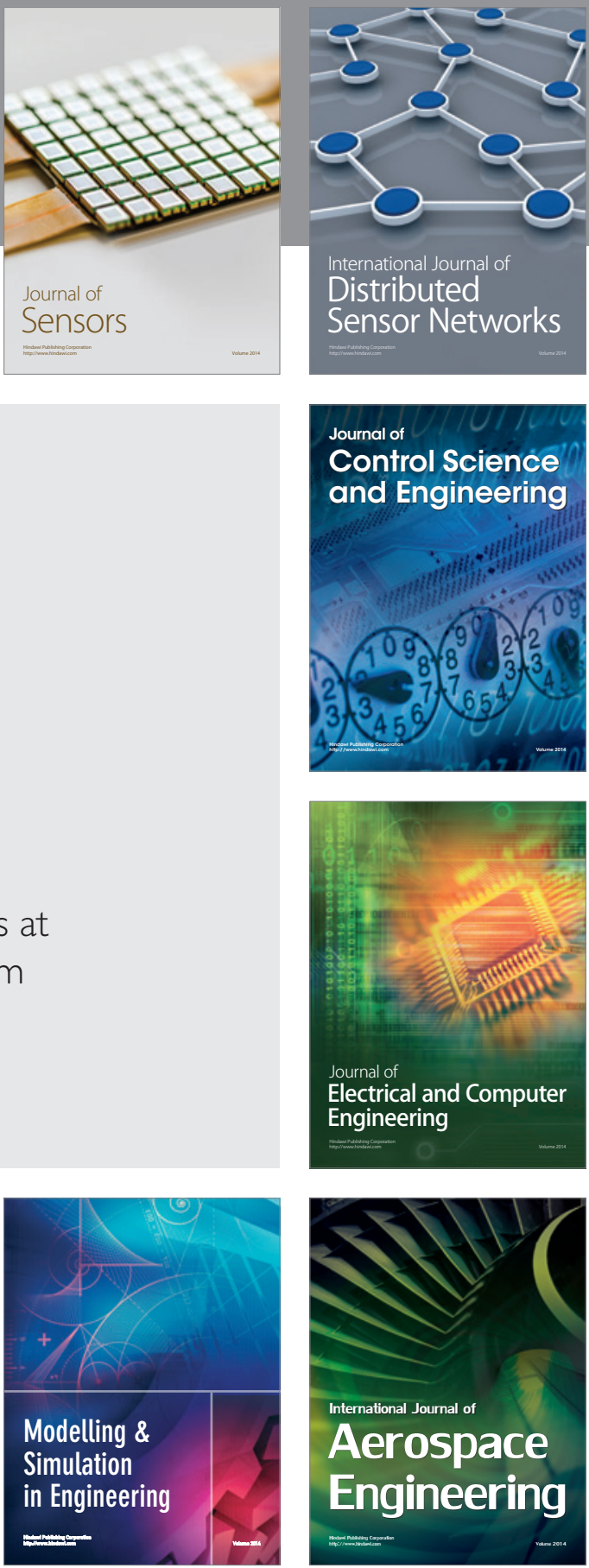

Journal of

Control Science

and Engineering
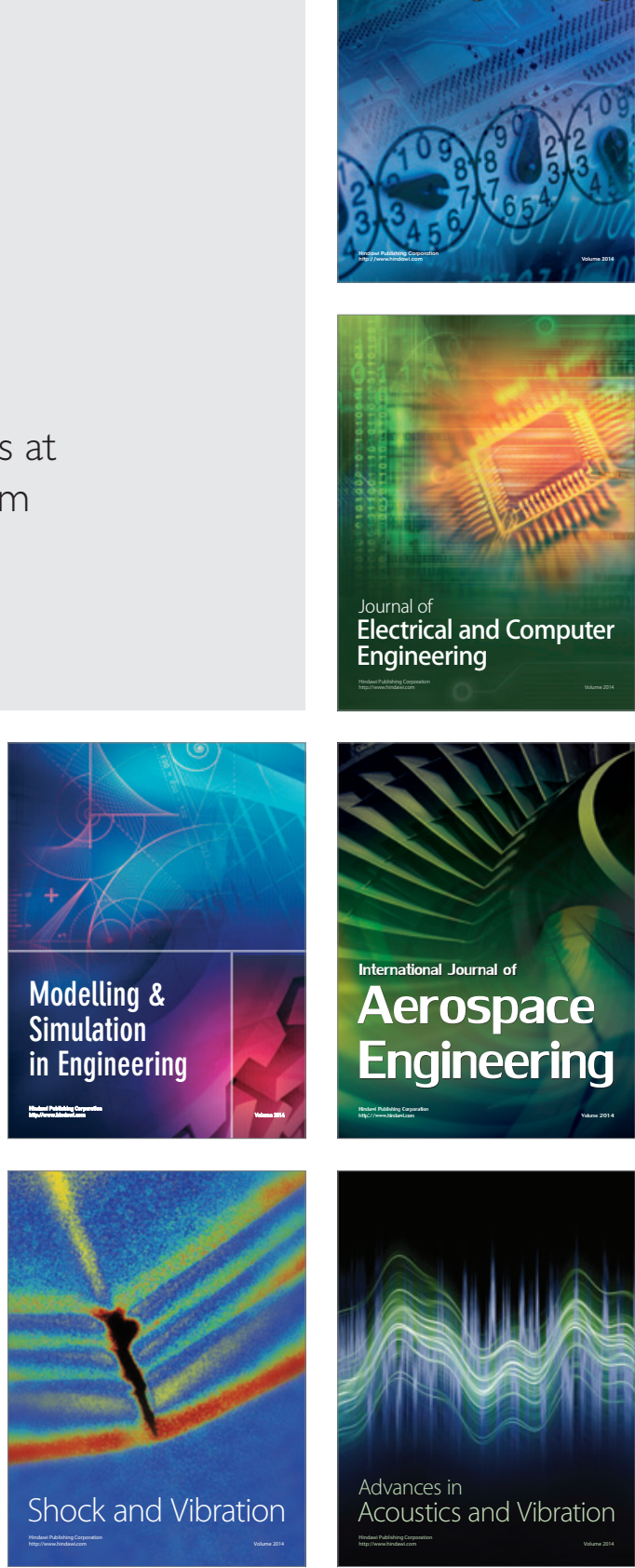